\title{
IDENTIFY AND RANK THE CHALLENGES OF IMPLEMENTING SUSTAINABLE SUPPLY CHAIN BLOCKCHAIN TECHNOLOGY USING THE BAYESIAN BEST WORST METHOD
}

\author{
Peide LIU $^{1 *}$, Ayad HENDALIANPOUR ${ }^{2}$, Mohammad HAMZEHLOU ${ }^{3}$, \\ Mohammd Reza FEYLIZADEH ${ }^{4}$, Jafar RAZMI ${ }^{5}$ \\ ${ }^{1}$ School of Management Science and Engineering, Shandong University of Finance and Economics, \\ 250014 Jinan, China \\ ${ }^{2}$ Soshianest Enterprise Miner, North Vancouver, Canada \\ ${ }^{3}$ Department of Management, University of Tehran, Tehran, Iran \\ ${ }^{4}$ Department of Industrial Engineering, Shiraz Branch, Islamic Azad University, Shiraz, Iran \\ ${ }^{5}$ School of Industrial Engineering, University of Tehran, Tehran, Iran
}

Received 20 July 2020; accepted 03 January 2021

\begin{abstract}
Globalization initiated the challenges in Supply Chain (SC) such as management and control. In this situation, Blockchain as a digital distributed ledger can guarantee clarity, tractability, and safety. Many case studies proved that we can use Blockchain Technology (BT) to solve global supply chain problems especially in smart contracts with their potential applications. BT is in its early period and it is hard to find supply chains that have successfully implemented this technology to track their sustainable actions. Therefore, it is worth studying about the role of customers, members, domestic, national, and international challenges that could resist implementing Blockchain and may affect SC sustainability. Accordingly, four categories of barriers to the use of BT are introduced which are inter-organizational, intra-organizational, technical, and external barriers. Then with Bayesian Best Worst Method, we ranked the BT barriers and the sub-barriers. The study illustrates the interconnection of these barriers and the priority of each element. The lack of business models and the best practices in implementing Blockchain technology is a challenge and it is important that practitioners acknowledge these barriers in the first steps.
\end{abstract}

Keywords: Supply Chain, Blockchain Technology, Bayesian Best Worst Method, sustainable.

JEL Classification: C11, D23, D81, D91.

\section{Introduction}

Contemporary Supply Chains (SCs) are naturally multifarious with multi-echelon, unconnected organizations geographically that struggle to serve customers (Carter et al., 2015; Hendalianpour et al., 2020b). It is practically impossible to assess data and manage risk in

${ }^{\star}$ Corresponding author. E-mail: peide.liu@gmail.com 
Supply Chain Networks (SCN) due to various controlling strategies, globalization, and different cultural and hominid behavior in this sophisticated network (Borgatti \& Li, 2009). Ineffective transactions, pilferage, fraud, and poor execution of SCs, results less trust and that is why SC members need better data exchange, and verifiability.

Traceability in many Supply Chain sectors, such as the agri-food industry, pharmaceutical, and high-value products is turning to be an increasingly pressing necessity and a basic differentiator (Skilton \& Robinson, 2009). Luxury and high-value products otherwise dependent on paper licenses and receipts may be readily lost or modified. Indeed, the absence of transparency in any item's supply value stops SC entities and clients from checking and validating that item's actual value (Hendalianpour et al., 2020a). Managing this traceability in the SC is further complicated by the costs engaged in managing intermediaries, transparency, and reliability. These risks and absence of transparency will harm strategic and reputational competitiveness. The triple-bottom-line notion, including stability of social, environmental, and business dimensions once handling SC, has determined sustainability (Hendalianpour, 2020; Skilton \& Robinson, 2009). The verification and confirmation that products, processes, and operations in the SC fulfill certain sustainability requirements and certifications is a significant strategic and competitive problem for SC sustainability (Busse et al., 2017). Given these concerns, the present SC information systems can support the data required for the timely source of services and products in a trustworthy manner in terms of clarity and strength should be determined. The answer to such complex issues is to improve SC transparency, durability, security, and process integrity. We believe that Blockchain Technology (BT) will be the solution to this issue. Novel technological applications and developments with BT notion make the objectives of the improvement more feasible technologically, organizationally, and economically (Flynn et al., 2016). BT provides global-scale transactions and procedure decentralization and disintermediation among different parties that integrates the features of decentralized trustless databases (Abhishek \& Divyashree, 2019).

There are some instances of first use that exemplify BT opportunities and issues such as Maersk and his corporation. In this situation, IBM stated that there could be billions in savings by having more precise and reliable landing bills connected to containers (Ellis et al., 2011). Although billions of savings are stated in implementing BT, owing to scaling problems, it is not evident that full execution was feasible. Originally, a Blockchain service provider, has attempted to incorporate BT into the seafood SC from a sustainable SC view. Validity and transparency of sustainable activities were critical in this situation (Adams et al., 2018). The prospective uses of Blockchain have been therefore well addressed in professional literature from environmental and socioeconomic perspectives. Despite its growing application in recent years, Blockchain may encounter many complications and obstacles in terms of execution and adoption in SC networks, as in the case of all potentially-disruptive schemes and technologies. We believe that Blockchain is in initial phases of development with different complications from technological, behavioral, organizational, or policy-oriented facets (Busse et al., 2017). For several years, these problems will be crucial in the discourse on scholarly literature. Today, the emerging practical problems determine and lead academic debates and discussions. These concerns need to be thoroughly and efficiently addressed. Regarding above indications, we can summarize the paper's contributions as follows: 
- Highlights the problems of and obstacles to Blockchain-based SCs and discusses the advantages and applications of implementing Blockchain in sustainable supply chain Considering uncertainty in demand, distribution and retail network.

- Using Bayesian Best-Worst Method to rank the challenges of implementing blockchain technology in sustainable supply chains.

- Rankings parameters affect the use of technology to Blockchina's sustainable SC enabling users to employ this technology in a sustainable SC in order to distinguish the obstacles with priority that help practitioners for proper planning and execution.

The remaining parts of the present article were structurally presented in the following manner: the first section introduces BT, applications in the Supply Chain Management (SCM) and discusses the advantages of Blockchain in terms of maintaining the sustainability of SC networks. Section second proposed the fifth segment categorizes the obstacles to implementing BT in SCs and supporting sustainability as four groups, then we present the research directions and recommendations in section three. Finally, we summarize the results and concludes practical recommendation.

\section{Literature review}

\subsection{Blockchain Technology}

BT is a shared private/public ledger or record database of digital actions performed and distributed amongst Blockchain participating agents (Kasten, 2019). Its background may be traced back to shared ledger technology. BT varies from the most current information systems by comprising four main features of decentralization, security, suitability (Saberi et al., 2019a), and smart execution (Figure 1). This original transaction is added as a new block to the chain when this transaction is authorized by the majority of nodes in the chain under pre-specified authorized guidelines. Several distributed nodes save a record of all transactions for the safety and tracing. Meantime, as BT critical element, the smart contract enables reliable transactions to be performed without the participation of the third parties.

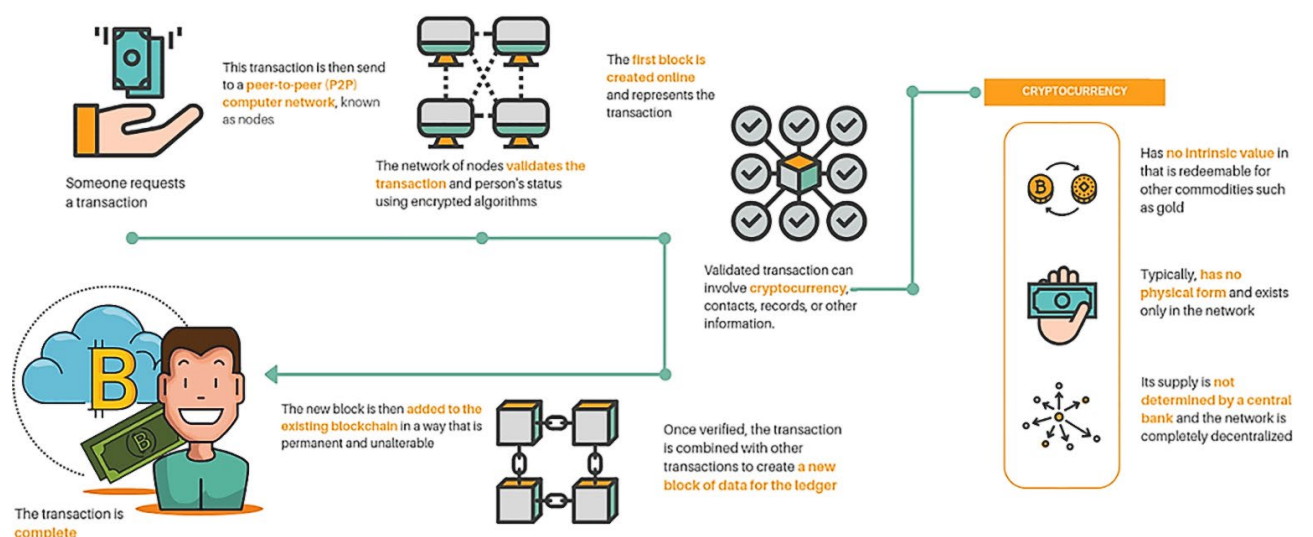

Figure 1. The data and transaction steps of BT 
BT's main difference from the present scheme of the internet lies in the fact that the internet was designed to transfer copies of stuff rather than values. Value is characterized in dealings documented in a distributed ledger and protected through the provision of a verifiable, time-stamped transaction record that offers safe and auditable data in Blockchains (Saberi et al., 2019a). Through a verification method, these transactions appear consistent with network consensus guidelines. As the novel validated record is added to Blockchain, multiple copies are generated in a decentralized model to produce the trusting chain.

Decentralization is a significant BT characteristic and is a check on every data contamination, hence raising its validity. Given the impossible removal of jointly-kept documents, a verified record of individual transactions is available to respondents via shared government or private documents (Underwood, 2016). Centralized databases are more vulnerable to crashing, corruption and hacking (Fanning \& Centers, 2016). In the network, usually there is no requirement to evaluate the reliability of the intermediary and other participants and data effortlessly is viewed and compared, trust is a major result of decentralization. This strategy requires no specific conducts on behalf of respondents; rather, the underlying technology ensures the integrity of the system even in untruthful or idle conditions (Tokar \& Swink, 2019). We all know that partakers can view ledgers and evaluate transactions. This characteristic ensures transparency while at the same time maintaining anonymity by maintaining cryptographic documents (Saberi et al., 2019a). A Blockchain can be generalized and applied to an agreed upon collection of guidelines that cannot be broken by anyone, neither the users nor the system operators.

The Blockchain layout that depends on the technology implementation can form public or private networks and ledgers (Schmidt \& Wagner, 2019). The scheme associated with the players of the network and guidelines for the Blockchain maintenance is also distinct. The parties understand each other in a personal or closed Blockchain and there is no anonymity. New roles such as certifiers in this situation confirm SC network respondents and maintain the private network. In other word, in an open or public Blockchain, cryptographic methods are implemented to allow customers to join the network and record their transactions with many anonymous customers (Manupati et al., 2020). A new generation of transactional applications supported by BT, which creates transparency, confidence and accountability, are managed through the so-called smart contract. A smart contract typically refers to a software program that stores policies and guidelines among parties for negotiating actions and terms which automatically confirms the realization of transactions and contractual terms (Manupati et al., 2020). The smart contract logic is implemented by a network of actors that agree on the execution result of the contract. The agreement runs the corresponding code as it receives a signal from another agreement or a network actor and; accordingly updates the ledgers if the contractual terms based on the private or public network are satisfied (Saberi et al., 2018). BT first became popular for handling the Bitcoin platform, a digital cryptocurrency (Saberi et al., 2019a). In addition to the digital currency, BT is a novel computation and information flow pattern with wide-ranging implications for future SC management and logistics development (Zijm et al., 2019). This point promoted us to the rest of this article; thus, we attempt to rank this domain's important indicators by Bayesian Best Worst Methods. 


\subsection{Blockchain-based SCs}

BT can disrupt the design, general management, operation and organization of SCs. The capacity of Blockchain to ensure data reliability, authenticity and traceability as well as intelligent contractual relationships in trustless settings necessitates significantly reconsidering SCs and their management. This section presented details the value and structure of BT and its application to the products and manufacture of SCs and potential fresh SC management components. How Blockchain works, is still accessible to analysis and growth within the SC framework presented in Figure 2.
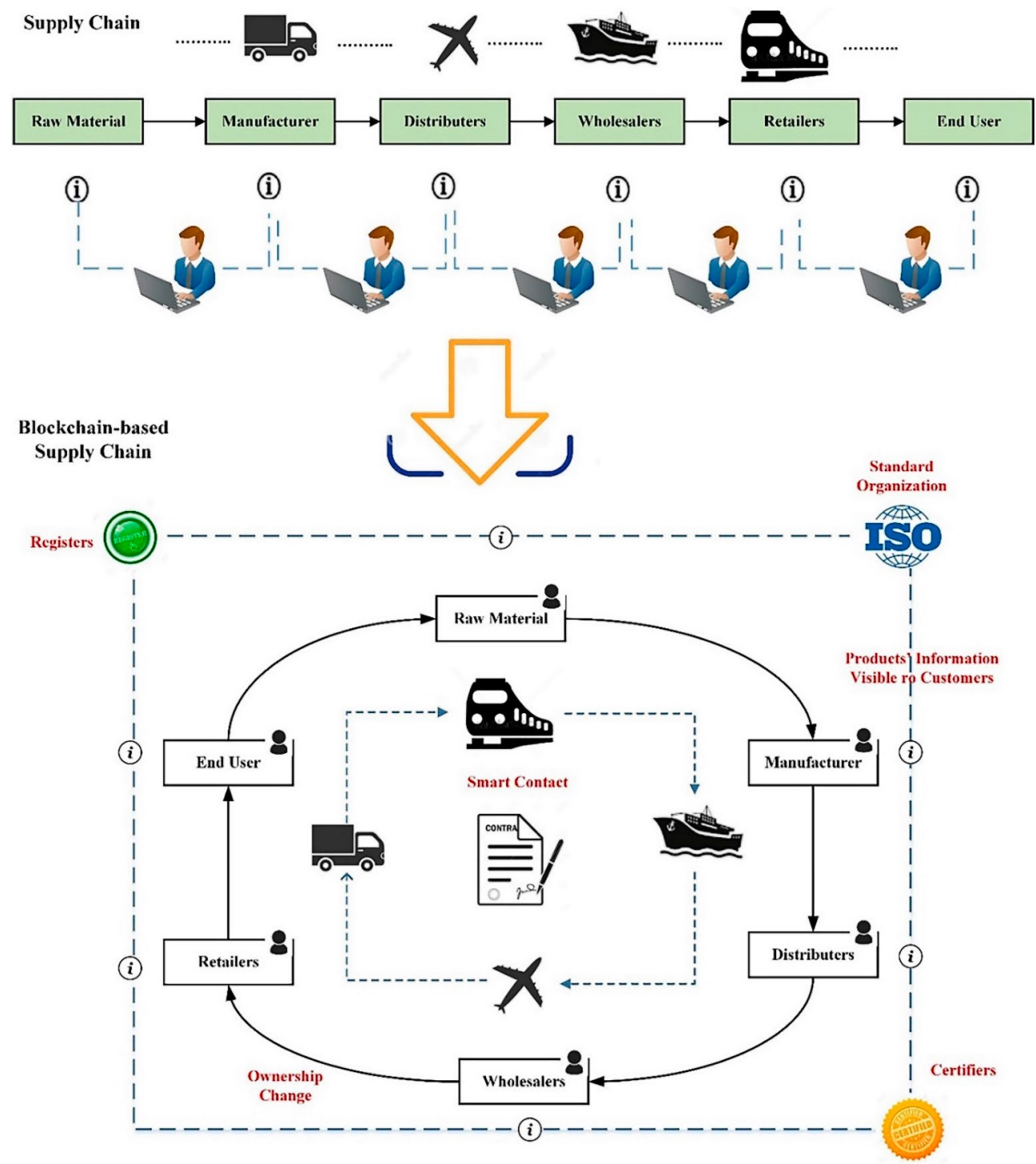

Figure 2. Supply Chain transformation 
In contrast to generally-public bitcoin and other monetary Blockchain applications, supply chain networks based on Blockchain may require a closed private permitted Blockchain with a limited number of actors. However, for more public relationship sets, the door can be yet open. Determining privacy levels constitutes an original alternative. Figure 2 shows a general outline of the conversion of a conventional to a Blockchain-based SC. Some of the four entities with a key role in a Blockchain-based SC may not be present in a conventional SC. Registrars offer distinctive identities to the network actors. Standards Organizations define norm systems such as sustainable SC Fairtrade as well as technological specifications and Blockchain policies. Certifiers authorize actors to get involved in the SC network. A certifier or auditor should certify actors such as customers and retailers as well as manufacturers to preserve the system confidence (Manupati et al., 2020).

There are also influences on the products of SC and the flows of material. All the relevant players can directly access a product profile using its individual digital Blockchain. Access can be limited using security processes in a way that a product can be accessed only by using the correct digital keys. The available collectable data include applicable standards to a product and its type and status (Tian, 2012). Physical goods are connected to the virtual identity of Blockchain through an identifier or data tag attached to a product (Saberi et al., 2019b). BT highlights and details at least five strategic dimensions of a product, including location, quantity or number, quality, ownership and nature (Schmidt \& Wagner, 2019). For instance, consumers may monitor accurate product data that would boost the confidence of clients in product features (Tian, 2012).

In a Blockchain-based SC, actor certification, as well as endorsement and procedures allowed to be reachable and required for implementation. Changes in the actor information may happen based on SC kind, status, and the cause determined by a smart contract. Without certain form of consensus, the players may not change the rules (Ølnes et al., 2017). Blockchain affects product management and the SC procedure, as well as financial dealings between various network sides (Hofmann et al., 2018). A major prospective benefit of Blockchain SC is disintermediating financial intermediaries, such as stock exchanges, payment networks, as well as money transfer services (Schmidt \& Wagner, 2019). This will make trading procedures more effective amongst partners. Supply Chain funding tools can decrease inefficiencies in SC economic flows and methods like dynamic discounting and reverse factoring (Tian, 2016); saving networks millions of dollars (Ivanov et al., 2019). Smart contracts can organize economic agreements and guarantee that the projects receive adequate resources and that is why everyone is compensated in a timely way (Hofmann et al., 2018). They offer a link between distinct currencies or blend them securely and in a timely manner from various sources in the worldwide SC (Eyal, 2017). While there may be a wide range of BT apps in the SC, they are concentrated on in terms of industry, governance, product, or service. Businesses and their SCs are pressured by customer, community, and regulatory to advance their SCs' sustainability and goods (Saberi et al., 2019b). This information led us to define in more detail potential SC implications by regarding the BT impact on sustainable SCs. 


\subsection{Blockchain and sustainable supply chains}

As transparent, immutable and reliable databases shared in a community, Blockchains can affect sustainable SC networks. Tracking social and environmental conditions with potential health, safety and environment hazards constitutes a major application of Blockchains (Adams et al., 2018). Blockchains created for carbon asset markets in China enabled companies to more efficiently generate carbon assets according to China's greenhouse gas emissions reduction stipulated in the Paris Agreement. A Blockchain-based SC can more effectively protect human rights and work procedures; for instance, a transparent history of a product reassures purchasers that the bought product was manufactured and delivered by ethicallyverified sources. In particular, a smart contract can autonomously track and control sustainable terms and regulatory policies and enforce or govern suitable corrections.

Sustainable SCs have gained momentous attention among practitioners and academics (Fahimnia et al., 2015). The company aspects of the SC are not only essential for sustainable SCs but expanding the emphasis on social and environmental aspects has made the SC more generalizable and holistic. As the promising features of BT, the environmental, financial and social bottom lines of sustainability constitute a panacea for this complexity. Supporting significant product and SC information, BT will support data collection, storage, and management as well. In this technological context, there is openness, reliability, neutrality, transparency, and security for all SC stakeholders and agents. Supply chains sustainability pressures are facing the food, beverage, and agriculture sector (Saberi et al., 2018). Blockchain may help SCs identify unethical vendors and counterfeit products as only approved actors can record all the data.

BT can immediately share any information alteration, so that products and procedures can be deployed quickly while minimizing human errors and transaction times. BT can guarantee data security and authenticity, lowering the cost of stopping intentional and capricious modification of information, increasing SC hazards and decreasing the efficiency of the company (Ivanov et al., 2019). Moreover, consumers and government now are calling for SC transparency. The competitive advantage of transparency was realized by pioneering businesses that leads to increased customer confidence to buy more and value the company financially.

BT has the ability to contribute to the sustainability of social SC. One way to build social sustainability for SC is to make data stable and immutable. A Blockchain can advise corrupt organizations, governments or individuals against unfairly confiscating people's property given the impossibility of altering the data without receiving the approval of the actors. In contrast to centralized conventional energy systems, a peer-to-peer BT-based network can decrease the need for the long transmission of electricity and therefore significantly save the energy wasted over long distances. The need for energy storage would also be reduced that saves its resources. BT-based energy platforms are available to decrease SC waste, including subcontract, Blockchain, and electric chain (Manupati et al., 2020).

Second, Blockchains could be applied to assure environmental friendliness of supposedly green goods. Green product data is often inaccessible and hard to check. For instance, in the sustainable Indonesian forest, Ikea has a woodcut desk product. The wood is tracked from the moment it is cut to the moment it is turned into the final product to ensure that the 
desk is made of this particular wood. This potentially-complicated process can, however, be managed using BT; for instance, BT was used to trace the origin of about 740 million acres of licensed forest in the world during the authorization of a forest certification program (Dolgui et al., 2018).

A further instance of the sustainability of environmental SC is a carbon tax. The carbon footprint of each item is hard to assess in traditional schemes. Tracing the footprint of a specific company's products becomes more straightforward with BT. It can assist in determining how much carbon tax business should be charged. The consumers can purchase a product of low-carbon footprint and avoid a large carbon footprint which is more costly. This extra data and customer or market pressure can lead companies to reassess and restructure their SC to decrease carbon emissions to satisfy buyers' requirement. By offering the basics for SC mapping and implementing low-carbon product design, manufacturing and transportation, BT can assist decrease carbon emissions in the product path (de Sousa Jabbour et al., 2018). The SC environmental analysis tool provided a framework for evaluating carbon emissions by individual entities engaged in SCs and product life-cycle (Saberi et al., 2019a).

Third, recycling can be enhanced by Blockchain. There may be no motivation for people and organizations to engage in recycling programs. BT-based economic incentives were utilized as cryptographic tokens to encourage Northern Europeans to deposit recyclables, including plastic containers, cans and bottles. In the meantime, the effect of different recycling programs is hard to monitor and compare. Blockchain allows information to be tracked to assess the effect of different programs. Social Plastic, for instance, is a BT-based project that aims to turn plastic into money and lessen plastic waste. Recycle to Coin is also an implementation of Blockchain that allows individuals to return plastic containers (Schmidt \& Wagner, 2019). The opportunities for closed-loop SCs of this sort of activity create Blockchain suitable for developing ideas like the circular economy.

Fourth, by enhancing the effectiveness of Emissions Trading Systems (ETS), Blockchain advantages the emissions trading process. By applying BT, fraud can be prevented because of Blockchain's fidelity and transparency. Hence, this creates a reputation-based scheme that solves the ineffectiveness of ETS and promotes all respondents to find a long-term alternative to the decrease of emissions because the financial advantages of excellent reputation encourage the participants (Gray et al., 2013).

Using the system of BT to govern SCs and manage their information is challenging, especially in sustainable networks. Disruptive technologies usually cause long-term and shortterm challenges (Manupati et al., 2020). Respondents in SCs should be therefore prepared to take it as an advantage rather than a threat, as it can challenge relationships over SC. These instances demonstrate the potential economic (sustainability), social and environmental effects that may be copied to Blockchain-enabled SCs.

\section{Barriers to applying BT to SCs}

We have described how BT can alter and improve the design, operations, and product flows of SC so far in this work. Here we are considering some of the issues about execution in this setting; particularly with regard to SCs, BT, and sustainability. Efficacious BT implementation to trace sustainable practices and manage SC procedures and products through SC starts with 
identifying problems and obstacles that need to be addressed. SC partners need to comprehend and schedule BT's acceptance and execution of these barriers.

In this part, pertinent literature, such as books, online database, journals, review papers, and conference papers, was studied to define multiple obstacles to BT implementation in general and sustainable SCs. Based on the literature on SC information systems, sustainable SCs, and BT, the obstacles were identified. These three main regions are the references that created the barriers list. Feedback from experts was also obtained to further validate the barrier list. The obstacles are summarized and divided into four major classifications of inter-organizational obstacles, intra-organizational obstacles, system-related obstacles, and external obstacles, taking into account organizational inner and external limitations in the adoption of novel technology.

\subsection{Intra-organizational barriers}

These set of obstacles arise from organizations inner operations. Support for top management is the main factor in implementing any SC procedures successfully. Some executives do not have long-run support and dedication to adopt new technologies and adhere to principles of sustainability. The management's lack of commitment prevents the integration of sustainability practices into SC procedures which would challenge allocations of resources (Rajeev et al., 2017) and financial choices. BT adoption needs investment in new data collection hardware and software that is expensive for organizations and network partners (Govindan \& Hasanagic, 2018).

It could be a challenge to lack the new organizational policies needed to clarify the use of BT. Adoption of BT may alter or transform existing organizational cultures (Schmidt \& Wagner, 2019). Organizational culture describes work culture rules, values and proper conduct in organizations. Furthermore, implementing BT in SC procedures needs novel roles, duties, and expertise to support various aspects of technology adoption (Mcivor, 2013).

Narrow technical expertise and knowledge about the use of BT act as an obstacle to this fresh technology being incorporated into the SC. Although Blockchain's interest in the technology industry is increasing, Blockchain's restricted number of apps and technical designers are a problem. BT is an information technology, which may be disruptive and needs that legacy systems be altered or replaced (Mcivor, 2013). Converting to new structures can alter the organizational culture or hierarchy and cause people and organizations to resist and hesitate. Theoretically, applying the Technology Acceptance Model (TAM), it is possible to predict and evaluate the applicability level of novel information technology concerning its utility and usability to people and organizations. Blockchain use may be assessed from TAM angle (Saberi et al., 2019a; Schmidt \& Wagner, 2019).

Organizations require embedding sustainability practices into their organizational vision and mission if they desire sustainable SCs to support a fresh information technology that is implemented throughout the SC network (Zhao et al., 2019). Practical plans are also required for achieving all-level sustainability all over SCs (Wang et al., 2019). The lack of conventional instruments, techniques, and indicators prevents the effective execution and measurement of sustainability practices for a certain organization in a Blockchain setting. Failure to implement BT business models and best practices is a challenge (Choi et al., 2020). 
Environmental regulations and rules are one of the primary drivers of sustainable actions in organizations. Organizations invest and seek to fulfill minimal sustainability requirements that may impede their innovativeness and creativity in implementing sustainable approaches at the same time (Choi et al., 2020). The requirements of customers for sustainable products and procedures can promote creativity in the application of sustainability. The lack of knowledge and readiness of customers to contribute to sustainable development is an obstacle to applying sustainability. Being unaware of green certification systems, consumers are therefore unwilling to contribute to recycling procedures and pay more for a sustainable product (Saberi et al., 2019b; Schmidt \& Wagner, 2019).

\subsection{Inter-organizational barriers}

This section identifies and primarily presents obstacles to the relationship of SC partners. SC management is primarily concerned with managing partnerships amongst partners in order to generate value for stakeholders (Schmidt \& Wagner, 2019). However, partnerships may be challenging, particularly once it comes to incorporating IT and sustainability methods. BT would promote the sharing of data via an SC. While transparency and verifiability of data are necessary to evaluate an SC's sustainability performance (Saberi et al., 2018), some organizations may suppose data as a competitive advantage that prevents them from sharing precious and critical data (Wang et al., 2019).

Lack of firm rules for sharing data ultimately impacts SC partners' cooperation. Lack of cooperation and efficient communication between SC partners having distinct and even conflicting operational priorities and objectives in mind distract sustainability and SC activities and the application of Blockchain to generating sustainable values (Rajeev et al., 2017). If SC partners are geographically detached with distinct cultures, communication difficulties would be worse (Hendalianpour et al., 2019).

Eventually, it is not a simple method to combine traditional SC procedures with sustainability methods. To promote sustainable methods, current techniques, designs, equipment, and procedures need to be enhanced (Zhao et al., 2019); for instance, facilities, materials and machinery are required to be updated to decrease waste, water pollution, energy consumption, carbon footprints and greenhouse gas emissions, which increases the costs of SCs. Similarly, for BT reasons, data collection mostly requires specific tools and equipment. The Internet of Things and Radio Frequency Identification are possible solutions to this problem.

\subsection{System-related barriers}

New IT instruments are required to implement BT and collect data for purposes of SC management (e.g., Internet of Things). For some SC members, this can be a challenge (Zhao et al., 2019). To benefit from the value-saving potential of integrated SCs, all the chain members should access their required data (Saberi et al., 2019b). Consequently, limiting access to technology to obtain real-time information in an SC is an obstacle to BT implementation. In its early growth phases, BT was considered immature in terms of transaction processing and 
scalability. The blocks growing in size and amount is actually a storage problem of Bitcoin for the real time handling of large-size data. Sophisticated cloud computing infrastructure and improved storage management are required to meet even greater information requirements expected in SC networks, which go beyond financial information and include process and practice associated information (Manupati et al., 2020).

Manipulating the data is a major cause for concern in SC networks. While adopting BT provides each SC network participant the chance to check transactions, collusion is still feasible by reaching a consensus among respondents (Tian, 2012). Also, data privacy and security concerns are challenges in BT use. Some study has discussed BT's safety challenge in the Bitcoin network, including hacks and assaults (Tian, 2016). While certain solutions to mitigate safety problems for Blockchain have been suggested, the effectiveness of these alternatives has not been assessed. However, BT is mainly connected with cryptocurrencies like Bitcoin and its malicious operations, the "dark web" reputation, which decelerates BT adoption as a whole. Another significant characteristic of BT is the immutability of data. It means that without consensus information may not be altered and deleted in Blockchain. This prevents information from being falsified and adulterated (Tian, 2016). However, people are still engaged in the application of this technology with the option of incorrect information recording. The Blockchain will always contain the scar of erroneous record even if the principal owners may revise the information and update it with further data (Saberi et al., 2018).

\subsection{External barriers}

This group of problems are caused by external governments, stakeholders and sectors as well as organizations that do not directly benefit economically from SC operations. Externallyapplied support and pressure on technological and sustainable procedures can cause organizations to involve them into their procedures. Obstacles to sustainability and advanced technology supporting mechanisms include the lack of appropriate industrial and governmental strategies and the desire to promote and direct secure and sustainable practices (Schmidt \& Wagner, 2019). The use of BT is still uncertain in government regulations and legislation. Negative policies adopted by different Bitcoin governments can actually cause problems for organizations and markets and lead to more extensively using company goals by Blockchains. Therefore, in order to generate sustainability value, governments, industries, communities, NGOs, and professional organizations should encourage BT. Furthermore, the uncertainty of demand for sustainable products and ambiguity of customer behavior can influence market competition and impede sustainability and BT integration. Organizations seek to ensure that they are compensated by customers for their investment in sustainable procedures, green products and novel technologies such as Blockchain (Schniederjans et al., 2020).

In order to develop the study agenda on BT implementation for SCs, we are proposing a range of study proposals based on the results of the SC implementation of BT. According to the above-mentioned contents, Table 1 summarizes the use of BT for SCs barriers. 
Table 1. Barriers to using Blockchain Technology in sustainable SCs (Saberi et al., 2018)

\begin{tabular}{|c|c|c|c|}
\hline Criterial & Index & Sub-criteria & Index \\
\hline \multirow{5}{*}{ Inter-organizational } & \multirow{5}{*}{ A1 } & $\begin{array}{l}\text { Lack of customer's awareness and tendency about } \\
\text { sustainability and BT }\end{array}$ & B1 \\
\hline & & $\begin{array}{l}\text { Problems in collaboration, communication and } \\
\text { coordination in the SC }\end{array}$ & B2 \\
\hline & & $\begin{array}{l}\text { Challenge of information disclosure policy between } \\
\text { partners in the SC }\end{array}$ & B3 \\
\hline & & $\begin{array}{l}\text { Challenge in integrating sustainable practices and } \\
\text { BT through SCM }\end{array}$ & B4 \\
\hline & & Culture difference of SC partners & B5 \\
\hline \multirow{5}{*}{ External } & \multirow{5}{*}{ A2 } & Lack of governmental policies & $\mathrm{C} 1$ \\
\hline & & Market competition and uncertainty & $\mathrm{C} 2$ \\
\hline & & Lack of external stakeholders' involvement & $\mathrm{C} 3$ \\
\hline & & $\begin{array}{l}\text { Lack of industry involvement in ethical and safe } \\
\text { practices }\end{array}$ & $\mathrm{C} 4$ \\
\hline & & Lack of rewards and encouragement programs & $\mathrm{C} 5$ \\
\hline \multirow{5}{*}{ System related } & \multirow{5}{*}{ A3 } & Security challenge & D1 \\
\hline & & Access to technology & D2 \\
\hline & & $\begin{array}{l}\text { Hesitation to adopt BT, due to negative public } \\
\text { perception }\end{array}$ & D3 \\
\hline & & Immutability challenge of BT & D4 \\
\hline & & Immaturity of technology & D5 \\
\hline \multirow{7}{*}{ Intra-organizational } & \multirow{7}{*}{ A4 } & Financial constraints & E1 \\
\hline & & Lack of management commitment and support & E2 \\
\hline & & $\begin{array}{l}\text { Lack of new organizational policies for using } \\
\text { technology }\end{array}$ & E3 \\
\hline & & Lack of knowledge and expertise & $\mathrm{E} 4$ \\
\hline & & Difficulty in changing organizational culture & E5 \\
\hline & & Hesitation to convert to new systems & E6 \\
\hline & & $\begin{array}{l}\text { Lack of tool for BT implementation in sustainable } \\
\text { SCs }\end{array}$ & E7 \\
\hline
\end{tabular}

\section{Methodology}

Due to the internal relationships in the criteria and the effectiveness and impact of the criteria from each other, also considering that the best worst method is able to calculate the effectiveness and effectiveness of the criteria based on the kernel method, so it has been selected in this study. Here, the ranking method is provided known as Bayesian Best Worst Method (BWM). The original BWM explained in section 3.1 and then the Bayesian BWM is provided in section 3.2 . 


\subsection{Best Worst Method}

BWM is a fairly novel multiple attribute decision making method (Rezaei, 2016). At the first the best and the worst criteria have been chosen and then the other criteria are compared with of these two criteria, to give an outline for the problem. Within such outline, the decision maker is contributed to offer more consistent pair-wise comparisons (Rezaei, 2016). Moreover, the specific BWM's structure results in two vectors comprising only figures hindering an essential distance problem related to using fractions in pair-wise comparisons (Salo \& Hämäläinen, 1997).

Some researchers proposed various ways for Group Decision-Making (GDM) with the BWM. Nevertheless, none of them offered a way to define the group's total weights in a probabilistic atmosphere. The following Stages are taken for the original BWM (Rezaei, 2016).

- Stage 1: The decision maker requires a group of decision criteria $C=\left\{c_{1}, c_{2}, \ldots, c_{n}\right\}$.

- Stage 2: The decision maker selects the worst $\left(c_{W}\right)$ and the best $\left(c_{B}\right)$ criteria from $C$.

- Stage 3: The decision maker performs pair-wise comparisons between $c_{B}$ and the other members of $C$ and determines the preference of the best criterion to the other criteria as $1-9$, where 1 denotes equally important and 9 significantly more important. The pair-wise comparison yielded the "Best-to-Others" vector of $A_{B}=\left\{a_{B 1}, a_{B 2}, \ldots, a_{B n}\right\}$, in which $a_{j W}$ implies the preference of $c_{j} \in C$ over the worst criterion $\left(c_{W}\right)$.

- Stage 4: The decision maker performs pair-wise comparisons between $c_{W}$ and the other members of $\mathrm{C}$ and determines the preference of the other criteria to the worst criterion as 1-9 as in stage 3. The pair-wise comparison yielded the "Others-to-Worst" vector of $A_{w}=\left(a_{w 1}, a_{w 2}, \ldots, a_{w n}\right)^{T}$.

- Stage 5: Achieving the optimal weights $w^{*}=\left(w_{1}^{*}, w_{2}^{*}, \ldots, w_{n}^{*}\right)$.

Considering $A_{B}$ and $A_{W}$, it is essential to compute a weight vector $w^{*}$. The weight vector lies is in the neighborhood of $w_{B} / w_{j}=a B_{j}$ and $w_{j} / w_{W}=a_{j} W$ for $j=1,2, \ldots, n$. Therefore, the maximum absolute differences $\left|\frac{w_{B}}{w_{j}}-a_{B j}\right|$ and $\left|\frac{w_{j}}{w_{W}}-a_{j W}\right|$ can be determined for all $j=$ $1,2, \ldots, n$. In addition, it is essential to fulfill the sum-to-one and non-negativity property of the weight vector. Consequently, the optimal weight vector $w^{*}$ can be obtained by the following optimization problem (Rezaei, 2016).

Depending on:

$$
\min -\max _{j}=\left\{\left|\frac{w_{B}}{w_{j}}-a_{B j}\right|,\left|\frac{w_{j}}{w_{W}}-a_{j W}\right|\right\} .
$$

$$
\sum_{j=1}^{n} w_{j}=1 \quad w_{j} \geq 0, \text { for all } j .
$$

Likewise, the following problem can determine the weight vector (Rezaei, 2016).

$$
\begin{aligned}
& \operatorname{Min} \xi \\
& \left|\frac{w_{B}}{w_{j}}-a_{B j}\right| \leq \xi \text { for all } j ;
\end{aligned}
$$




$$
\begin{aligned}
& \left|\frac{w_{j}}{w_{W}}-a_{j W}\right| \leq \xi \text { for all } j ; \\
& \sum_{j=1}^{n} w_{j}=1 \quad w_{j} \geq 0 \text {, for all } j .
\end{aligned}
$$

The following Consistency Ratio (CR) was utilized to assess the reliability of the optimal weights and investigate the match between input pair-wise comparisons and their corresponding output weights:

$$
C R=\frac{\xi^{*}}{C I}
$$

where Constancy Index (CI) represents a constant per $a_{B W}$ obtained from Table 2 and $\xi^{*}$ the optimal impartial value of model (2).

Table 2. Values of CI

\begin{tabular}{|c|c|c|c|c|c|c|c|c|c|}
\hline$a_{B W}$ & 1 & 2 & 3 & 4 & 5 & 6 & 7 & 8 & 9 \\
\hline $\mathrm{CI}$ & 0.00 & 0.44 & 1.00 & 1.63 & 2.30 & 3.00 & 3.73 & 4.47 & 5.23 \\
\hline
\end{tabular}

CR lies between 0 and 1 , which 0 denoting complete consistency. In other words, the higher the $\mathrm{CR}$, the lower the consistency of the pair-wise comparison.

\subsection{Probabilistic interpretation BWM}

The best-worst framework was used to present a Bayesian hierarchical model and determine optimal weights for a set of criteria in terms of multiple preferences of the decision maker.

\subsubsection{Group Decision-Making: A joint probability distribution}

Let the $k^{\text {th }}$ decision maker, $k=1, \ldots, K$, calculates criteria $c_{1}, \ldots, c_{n}$ while offering vectors $A_{B}^{k}$ and $A_{W}^{k}$. The set of all the $K$ decision maker vectors were shown as $A_{B}^{1: k}$ and $A_{W}^{1: k}$. Henceforth, superscript ${ }^{1: k}$ represent the overall vectors of the base. The optimal weight was also shown as $w^{a g g}$, which can be estimated with different auxiliary variables. In particular, $w^{\text {agg }}$ was calculated in terms of the optimal $K$ decision makers' weights obtained by $w^{k}, k=1, \ldots, K$. Therefore, $w^{a g g}$ and $w 1: K$ would be instantaneously computed by the proposed Bayesian model. Before performing any statistical analyses, deriving the joint probability distribution of all the random variables based on the available data is essential. $A_{B}^{1: k}$ and $A_{W}^{1: k}$ were obtained in the GDM of the BWM. As a result, $w 1: K$ and $w^{a g g}$ could be estimated. Accordingly, one should obtain the following joint probability distribution:

$$
P\left(w^{a g g}, w^{1: K} \mid A_{B}^{1: k}, A_{W}^{1: k}\right) .
$$

By computing the probability in (4), then utilizing the following probability rule, each separate variable's probability can be calculated.

$$
P(x)=\sum_{y} P(x, y)
$$

in which $x$ and $y$ show arbitrary random variables. 


\subsubsection{Bayesian hierarchical model}

The independence and conditional independence of the variables were first determined to develop the Bayesian model. In Figure 3, the graphical models are represented equivalent to the proposed model. The variables were shown as the graph nodes. The input rectangles to the original BWM were considered to be the observed variables. The variables as the circular nodes should be estimated. In addition, arrows indicate that the node originally depends on the node at the other end. In other words, the value of $w_{k}$ relies on $A_{W}^{k}$ and $A_{B}^{k}$, and the value of $w^{a g g}$ depends on $w_{k}$ as well.

The plate covering a group of variables indicated that the equivalent variables were repeated for individual decision makers and $w^{\text {agg }}$ do not exist in the plate as merely one $w^{a g g}$ exists for decision makers. According to Figure 3, the provisional independence between different variables is clear. For example, $A_{W}^{k}$ is not dependent on $w^{a g g}$ given $w_{k}$, i.e.

$$
P\left(A_{W}^{k} \mid w^{a g g} \cdot w^{k}\right)=P\left(A_{W}^{k} \mid w^{k}\right) .
$$

Given all independences among various variables, the Bayes rule should be applied to the joint probability (4) as follows:

$$
\begin{aligned}
& P\left(w^{a g g}, w^{1: K} \mid A_{B}^{1: k}, A_{W}^{1: k}\right) \propto P\left(A_{B}^{1: k}, A_{W}^{1: k} \mid w^{a g g}, w^{1: K}\right) P\left(w^{a g g}, w^{1: K}\right)= \\
& P\left(w^{a g g}\right) \prod_{k=1}^{K} P\left(A_{W}^{k} \mid w^{k}\right) \cdot P\left(A_{B}^{k} \mid w^{k}\right) \cdot P\left(w^{k} \mid w^{a g g}\right) .
\end{aligned}
$$

The final equality was achieved using the conditional independence and the probability chain rule of different variables and the decision maker offers were found to self-reliantly offer their preferences. The dependence of estimating parameters in Equation (7) on the estimates of the other variables suggested a chain between different elements and confirmed the hierarchical nature of the model. Now, one should determine the distributions of each parameter in Equation (7). $A_{B}$ and $A_{W}$ were found to be modeled using the multinomial distribution in a way that it provided the BWM's fundamental issue. Only one alteration can be found between $A_{W}$ and $A_{B}$ as the former includes the preference of the best over all the other criteria, while the preferences of all the criteria over the worst were represented with the latter. Therefore, they can be modeled as (8):

$$
\begin{aligned}
& A_{B}^{k} \mid w^{k} \sim \operatorname{multinomial}\left(1 / w^{k}\right) ; \\
& A_{W}^{k} \mid w^{a g g} \sim \operatorname{multinomial} w^{k} .
\end{aligned}
$$

Considering $w^{a g g}$, it can be expected that every and each $w_{k}$ is in its proximity. To this end, we reparametrize the Dirichlet distribution with respect to its mean and a concentration parameter. The models of $w_{k}$ given $w^{\text {agg }}$ are: 


$$
w^{k} \mid w^{a g g} \sim \operatorname{Dir}\left(\gamma \times w^{a g g}\right),
$$

where $w^{a g g}$ shows the mean distribution and $\gamma$ represents the concentration element.

According to Equation (9), the weight vector $w_{k}$ related to individual decision makers should lie in the neighborhood of $w^{a g g}$ as it shows the distribution mean, and the non-negative parameter $\gamma$ controls their closeness. It should be modeled the concentration parameter as well through a distribution. The gamma distribution is consistent choice satisfying the non-negativity restraints, i.e.:

$$
\gamma \sim \operatorname{gamma}(a, b)
$$

where $a$ and $b$ show the gamma distribution's shape limits. We ultimately find the prior distribution over $w^{a g g}$ through a vague Dirichlet distribution with the parameter $a=1$ as.

$$
w^{a g g} \sim \operatorname{Dir}(\alpha)
$$

Since a closed-form solution is not supplied by specified model, it is essential to use Markov-chain Monte Carlo (MCMC) methods (Kass et al., 1997) to calculate the later distribution. Just Another Gibbs sampler (JAGS) was employed as an effective probabilistic language proposed to date in the MCMC sampling (Plummer, 2004) for sampling and calculating the subsequent estimating parameters defined in Equation (7). The strengths of the model include the subsequent distribution of the weights for individual decision makers and

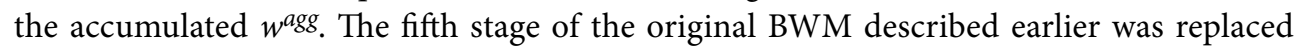
with the proposed Bayesian model. The optimization problem was indeed replaced with a probabilistic model with the same inputs. Though, more information is provided by the proposed model considering the confidence of the association between each criteria pair. By developing a novel Bayesian test in terms of the approached distribution from the model, more information is obtained, which was clarified in the next section.

\subsection{Credal ranking}

According to the modus operandi in Multiple-Criteria Decision-Making (MCDM), a criterion is more important than another in case its weight or the mean weight for the GDM is higher than that of the other criterion. Assume three criteria, i.e., $c_{1}, c_{2}$ and $c_{3}$, with the weight vector of $w=[0.49,0.50,0.01]$. According to the MCDM, $c_{2}$ is the most important in these criteria. Pairwise comparison is, however, inadequate for determining the superiority confidence. If the weight vector represents the preferences of a group of decision makers, this is even further imperative. Different ranking outline have been existed so far like interval-oriented ranking, fuzzy ranking, and ranking in terms of Grey Relational Analysis (GRA) (Yatsalo \& Martinez, 2018). Now, the credal ranking notion is presented able to adjust the grade by which one criterion is more important than another is. The confidence of the relationships among different criteria can be measured using the weights subsequent distribution. The alteration between other rankings and the credal ranking outlines is that in the credal ranking a confidence is calculated in terms of one distribution, indeed, the Dirichlet distribution of $w^{a g g}$. Other ranking approaches, however, normally preserve 2 intervals/numbers and seeks to discover the relative importance of one. The credal ordering was initially defined as the building block of credal ranking. 
Definition 1: A credal ordering of $\mathrm{O}$ is explained as the following for a pair of criteria $c_{i}$ and $c_{j}$ :

$$
O=\left(c_{i}, c_{j}, R, d\right),
$$

where $R$ represents the association of criterion $c_{i}$ with $c_{j}$, i.e., $\langle$,$\rangle , or =$ and $d \in[0,1]$ the relationship confidence.

Definition 2: For a set of criteria $C=\left\{c_{1}, c_{2}, \ldots, c_{n}\right\}$, credal ranking refers to a group of credal orderings that include all pairs of $\left(c_{i}, c_{j}\right)$ for $c_{i}, c_{j} \in C$.

Decision makers with more information can be obtained by the credal ordering confidence able to considerably enhance their decisions. A novel Bayesian test was developed to obtain the credal ordering confidence. The examination was estimated on the subsequent distribution of $w^{a g g}$. Equation (13) was utilized to calculate the confidence for the superiority of $c_{i}$ to $c_{j}$.

$$
P\left(c_{i}>c_{j}\right)=\int I_{\left(w_{i}^{a g g}>w_{j}^{a g g}\right)} P\left(w^{a g g}\right),
$$

where $P\left(w^{a g g}\right)$ shows the subsequent distribution of $w^{a g g}$, while $I$ is 1 in case the condition in the subscript takes and otherwise 0 . The specimens found via the multi-criteria decision making can estimate this integration. The confidence is calculated as follows using the $Q$ samples obtained from the later distribution:

$$
\begin{aligned}
& P\left(c_{i}>c_{j}\right)=\frac{1}{Q} \sum_{q=1}^{Q} I\left(w_{i}^{a g g_{q}}>w_{j}^{a g g_{q}}\right) ; \\
& P\left(c_{j}>c_{i}\right)=\frac{1}{Q} \sum_{q=1}^{Q} I\left(w_{j}^{a g g_{q}}>w_{i}^{a g g_{q}}\right) .
\end{aligned}
$$

where $w^{a g g}$ is the $g^{\text {th }}$ sample of $w^{\text {agg }}$ from the MCMC specimens. Therefore, for each pair of criteria, the confidence can be computed for the superiority of one criterion to another. It is possible to alter credal ranking only into the conventional ranking. Incidentally, it is obvious that $P\left(c_{i}>c_{j}\right)+P\left(c_{j}>c_{i}\right)=1$. Thus, $c_{i}$ is more superior than $c_{j}$ if and only if $P\left(c_{i}>c_{j}\right)>0.5$. Consequently, using a 0.5 threshold to the credal ranking can reach the traditional ranking of criteria.

\section{Case study results}

In this section, we analyze a real-world example using the Bayesian BWM. Also, the credal ranking is calculated and visualized using five weighted directed graphs (Figures 4 to 8). The data in this case study was gathered from 50 decision-makers. These decision-makers are comprised of 25 academic people and 25 experts from different companies who were familiar with BT and had experiences in sustainable SCM. Among the academic experts of universities, seven DM obtained Ph.D., eight DM got master, and the rest have bachelor degree. Also, eight experts had more than 15 years' experience in this field and the remaining have experience between 10 to 15 years and the experience. Out of the total number of decision-makers, 34 persons are men and 16 persons are women. 
Table 3 and Figure 4 show the results of the weights and importance of each of the four main categories of attributes investigated in this study. According to experts, the attributes $\mathrm{A}_{4}$ (Intra-organizational) and $\mathrm{A}_{2}$ (External) are the first and second ranks of importance respectively in both BMW and Bayesian BMW methods. One of the reasons that these attributes were selected as the most important is these barriers are rooted in the internal activities of organizations. High-level management support is a key factor in the successful implementation of any SC action. However, some managers cannot have long-term commitment and support to apply new technology and stay loyal to sustainable values. Lack of management awareness and commitment in the SC will challenge financial decisions and resource allocations. Accepting BT also requires investment in new hardware and software to gather information, which is costly for organizations and network partners. The lack of new organizational policies needed to explain the use of BT can be a challenge. Using BT may change or transform current organizational cultures. Organizational culture describes the guidelines for cultures, values, and appropriate behavior within organizations. Also, the use of BT in the SC process requires new roles, responsibilities, and expertise to support various aspects of technology use.

Table 4 and Figure 5 show the weights and importance of the sub-criteria of inter-organizational barriers. Based on Table 4 the sub-criteria $\mathrm{B}_{3}$ (Challenge of information disclosure policy between partners in the $\mathrm{SC}$ ), $\mathrm{B}_{2}$ (Problems in collaboration, communication and coordination in the $\mathrm{SC}$ ) and $\mathrm{B}_{4}$ (Challenge in integrating sustainable practices and $\mathrm{BT}$ through SCM) are the most important criteria in this category. This classification mainly identifies and introduces barriers to SC partnerships. In essence, SCM is about managing relationships between partners to create value for shareholders. Thus, relationships between partners can be challenging, especially when it comes to integrating information technology and sustainable action. BT will facilitate information sharing through a SC. Although transparency and verifiability of information is a requirement for evaluating the sustainability performance of a SC, some organizations may view information as a competitive advantage that makes them reluctant to share valuable and vital information. Doubting the disclosure of information from some partners may limit the full benefits of using BT and prevent the successful implementation of this technology. Different privacy policies related to the use and distribution of data and information in SCs may lead to new challenges for data sharing between partners. Because of the transparency of information in BT, information sharing policies and rules must be defined and managed within the SCN. The lack of strong rules for information sharing ultimately affects cooperation among SC partners. The lack of effective cooperation and communication between SC partners with goals and priorities disrupts even contradictory sustainability and the operation and implementation of the SC Blockchain to create sustainable values.

Table 5 and Figure 6 show the weights and importance obtained from the criteria of the external barriers. As can be seen from Table 5, the criterion $\mathrm{C}_{3}$ (Lack of external stakeholders' involvement) has the highest weight and importance according to experts. The reason for the importance of these criteria in foreign barriers is that foreign shareholders, industries, institutions, and governments do not directly benefit from the activities of the SC economically. External pressures and support to implement technological and sustainable actions can motivate organizations to integrate them with their processes. The lack of a proper industrial and governmental policy and willingness to direct and support sustainable and secure action is a barrier to achieving sustainable and technologically advanced sustainability mechanisms. 
As a result, governments, NGOs, industries, communities, and professional organizations need to upgrade BT to create sustainable value. In addition, the uncertainty of demand for sustainable products and ambiguity in customer behavior may affect market competition and prevent the integration of sustainability and BT. Organizations need to ensure that their investment in green products, sustainable processes, and new technology such as Blockchain, will compensate by their customers.

New IT tools are needed to implement BT and data collection to ensure the SC management process (for example, the Internet of Things). This could be a change for some participants in the SC. Therefore, all participants in a chain need to have access to the information needed to take advantage of opportunities to save value in an integrated SC. Therefore, the limitation of access to technology to obtain real-time information in a SC is a barrier to the implementation of BT. Therefore, according to Table 6 and Figure 7, the results of this table confirm the improvement of the overall structure of the stable SC network, forcing them to use new technologies such as Blockchain. Accordingly, in Table 6, the criterion $\mathrm{D}_{4}$ (Immutability challenge of BT) has the highest importance and coefficient according to the opinion of experts.

Table 3. Comparison between BWM and Bayesian BWM of applying BT to SCs barriers

\begin{tabular}{|l|c|c|c|c|}
\hline & A1 & A2 & A3 & A4 \\
\hline BWM & 0.1282 & 0.2799 & 0.1931 & 0.3989 \\
\hline Bayesian BWM & 0.1265 & 0.2791 & 0.1943 & 0.4002 \\
\hline
\end{tabular}

Table 4. Inter-organizational

\begin{tabular}{|l|c|c|c|c|c|}
\hline & B1 & B2 & B3 & B4 & B5 \\
\hline BWM & 0.2079 & 0.1969 & 0.2029 & 0.1986 & 0.1755 \\
\hline Bayesian BWM & 0.2068 & 0.1987 & 0.2195 & 0.1993 & 0.1757 \\
\hline
\end{tabular}

Table 5. External

\begin{tabular}{|l|c|c|c|c|c|}
\hline & C1 & C2 & C3 & C4 & C5 \\
\hline BWM & 0.2031 & 0.1951 & 0.2400 & 0.1714 & 0.1851 \\
\hline Bayesian BWM & 0.2020 & 0.1971 & 0.2366 & 0.1743 & 0.1899 \\
\hline
\end{tabular}

Table 6. System related

\begin{tabular}{|l|c|c|c|c|c|}
\hline & D1 & D2 & D3 & D4 & D5 \\
\hline BWM & 0.2021 & 0.1798 & 0.2067 & 0.2137 & 0.1998 \\
\hline Bayesian BWM & 0.1999 & 0.1854 & 0.2031 & 0.2137 & 0.1980 \\
\hline
\end{tabular}

Table 7. Intra-organizational

\begin{tabular}{|l|c|c|c|c|c|c|c|}
\hline & E1 & E2 & E3 & E4 & E5 & E6 & E7 \\
\hline BWM & 0.1351 & 0.1382 & 0.1391 & 0.1195 & 0.1497 & 0.1636 & 0.1567 \\
\hline Bayesian BWM & 0.1338 & 0.1363 & 0.1390 & 0.1212 & 0.1485 & 0.1633 & 0.1578 \\
\hline
\end{tabular}




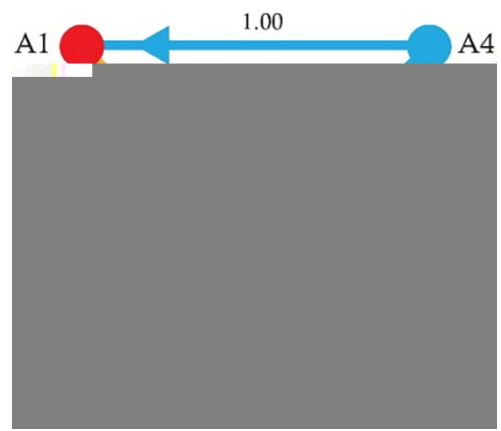
A1 Inter-organizational barriers
A2 External barriers
A3 System related barriers
A4 Intra-organizational barriers

Figure 4. The visualization of the credal ranking for main barriers

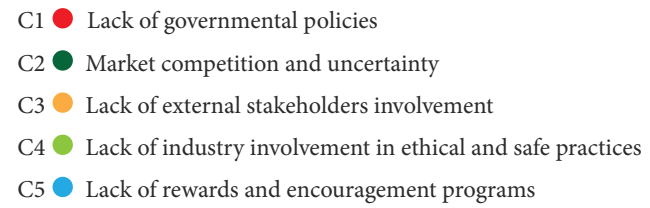

Figure 5. The visualization of the credal ranking external barriers

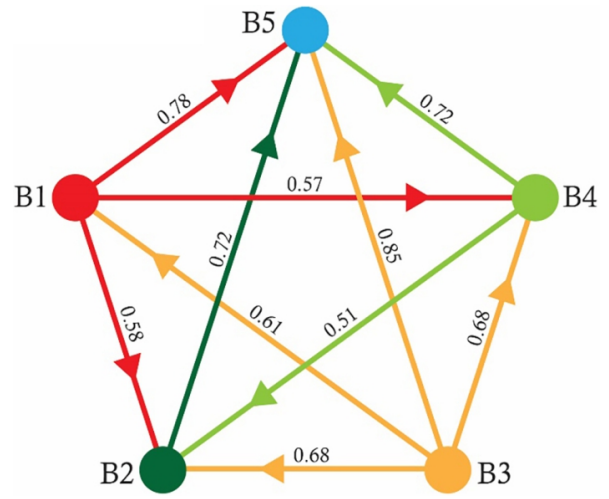
B1 Lack of customers awareness and tendency about sustainability and blockchain technology
B2
Problems in collaboration, communication and coordination in the supply chain
B3 Challenge of information disclosure policy between partners in the supply chain
B4 Challenges in integrating sustainable practices and blockchain technology through SCM

B5 Cultural differences of supply chain partners

Figure 6. The visualization of the credal ranking for inter-organizational barriers

The lack of new organizational policies needed to explain the use of BT can be a challenge. The use of BT may change or transform current organizational cultures. Also, the use of BT in the SC process requires new roles, responsibilities, and expertise to support various aspects of technology use. Accordingly, experts allocate the highest weight and importance 
to the attributes of the category $\mathrm{E}_{6}$ (Hesitation to convert to new systems) shows in Table 7 and Figure 8. Limited expertise and technical knowledge of using BT act as a barrier to the application of this new technology in the SC. However, the growing interest in Blockchain in the technical market, the limited number of applications, and the BT developers are matters of concern. BT is an information technology that can be disruptive and requires modification or replacement of survivor systems. Transforming to new systems may change organizational culture or hierarchy and lead to resistance and uncertainty from individuals and organizations. If organizations want to have sustainable SCs with the support of a new information technology that is used by the entire SCN, they need to consider sustainable actions on their mission and organizational version. Lack of standard tools, methods, and criteria of successful implementation and measurement of sustainability measures in a Blockchain environment will lead to creating an unsuccessful organization.
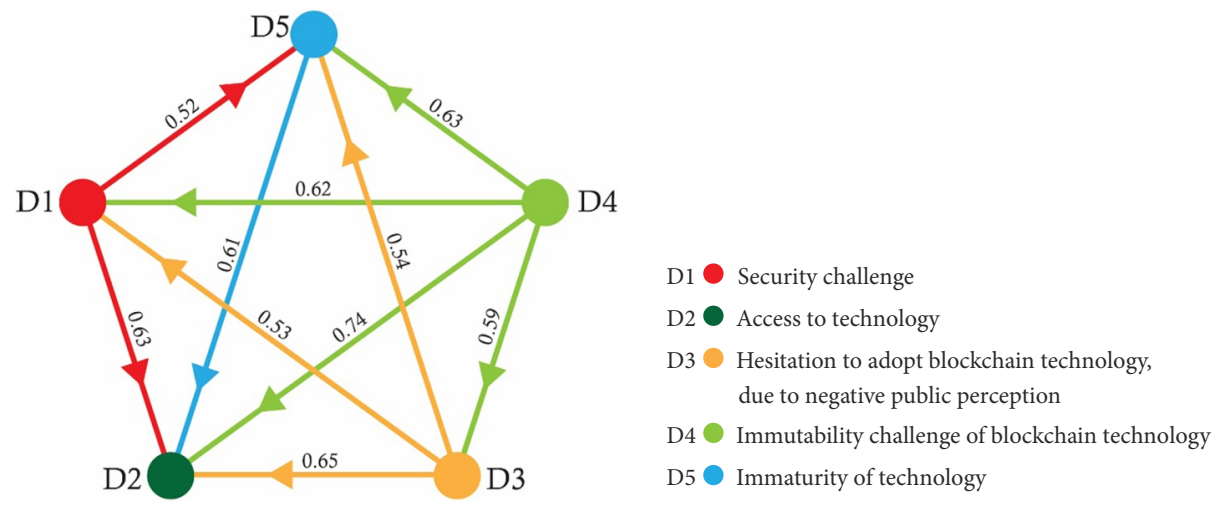

Figure 7. The visualization of the credal ranking for system related barriers

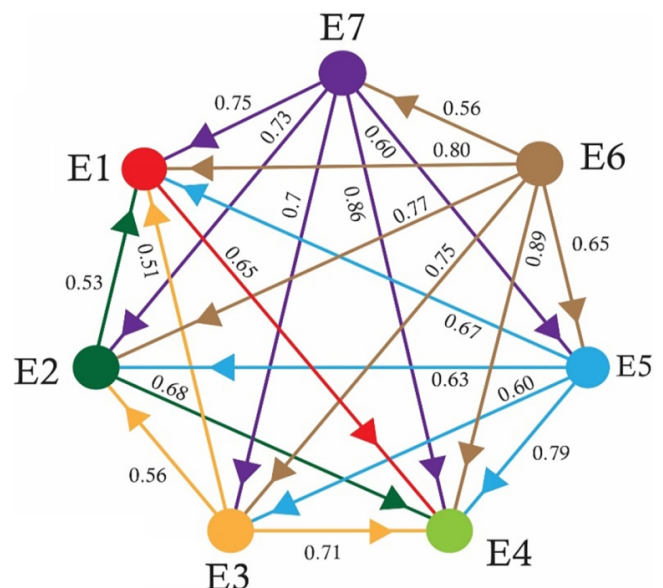

\footnotetext{
E1 Financial constraints

E2 Lack of management commitment and support

E3 Lack of new organizational policies for using technology

E4 Lack of knowledge and expertise

E5 Difficulty in changing organizational culture

E6 Hesitation to convert to new systems

E7 Lack of tool for blockchain technology implementation in sustainable supply chains
}

Figure 8 . The visualization of the credal ranking for intra-organizational barriers 


\section{Conclusions}

The innovative applications of Blockchain have been considered in literature from socio-economic and environmental perspectives. This study firstly specifies the barriers of Blockchain based on SCs and brings up the benefits and applications of BT. It is illustrated that there are four barriers in implementing BT. Afterward, the new MADM method (Bayesian Best Worst Method) have been applied for ranking the barriers related to the implementation of Blockchain in sustainable SC. Accordingly, ranking these barriers affects the application of technology to Blockchain biased sustainable SC. This ranking enables practitioners to have more appropriate planning for a sustainable SC based Blockchain.

Based on published literature, we reviewed the list of criteria and sub-criteria that affect the implementation of BT in the supply chain. Then, based on the opinions of experts in this field, we obtained the weight and importance of each criterion in different categories. In conclusion, we found out that the most important criteria for the successful implementation of BT in the sustainable supply chain will be inter-organizational barriers and external barriers. As shown in Table 3 and Figure 4, the criteria $\mathrm{A}_{4}$ (Intra-organizational) and $\mathrm{A}_{2}$ (External) were the two most important criteria for experts in terms of weight, respectively. It is clear from Figure 4 that $\mathrm{A}_{4}$ is associated with all three criteria. Also, considering the importance and credal ranking, we found that criterion $\mathrm{A} 4$ has the most connection with the criteria $A_{1}$ (inter-organizational) and $A_{3}$ (system-related) with the value of one. The results confirm that if organizations intend to have sustainable supply chains with the support of a new information technology used by the entire supply chain network, they should put sustainable actions in their mission and organizational version. Active plans are also needed to advance sustainability at all levels of the organization and the entire supply chain.

The lack of standard tools, methods, and indicators prevents successful implementation and measurement of sustainability actions in a Blockchain environment for a specific organization. We all know that BT is in its early stages, and it is difficult to find supply chains that have successfully implemented this technology to track their sustainable actions. The lack of business models and the best practices in implementing BT is a challenge. One of the main drivers in this regard is the use of sustainable actions in organizations, regulations and environmental laws. Organizations are investing and searching to meet the minimum standards of sustainability, which can simultaneously prevent their creativity and innovation in implementing sustainable methods. A stimulus that can promote creativity in sustainable development is customer demand for sustainable products and methods. Lack of customer awareness and disinterest in helping sustainable development is a barrier to sustainable development. In this case, some customers do not understand green plans and do not want to help with recycling processes or pay more for sustainable products. Also, to overcome external barriers, first, it is better to review the most important issues (as shown in Table 1). Then, for smoothing the supply chain trend, we need to use the suitable clustering method, BT barriers, and an effective understanding of new technology in supply chain networks and build sustainable aspects. These factors have not been experimentally tested or confirmed; however, the recommended frameworks and factors in this study provide a starting point for the successful implementation of BT in the supply chain. 


\section{Acknowledgements}

This paper is supported by the National Natural Science Foundation of China (Nos. 71771140, 71471172 and 71271124), Project of cultural masters and "the four kinds of a batch" talents, the Special Funds of Taishan Scholars Project of Shandong Province (No. ts201511045), Major bidding projects of National Social Science Fund of China (19ZDA080).

\section{References}

Abhishek, N., \& Divyashree, M. S. (2019). Environmental friendly supply chain management - a perception analysis. Journal of Supply Chain Management, 8(2), 45-51. http://search.ebscohost.com/ login.aspx? direct=true\&profile $=$ ehost $\&$ scope $=$ site\&authtype $=$ crawler\&jrnl $=22771387 \& A N=13876$ 5370\&h=FaYhWygq97nSk1yIoGvL9M5qleVLOrS57gcbuPk4sJCHM7G2WyNDc9\%2BRkFO\%2F0 PR1OUPsr\%2FwfWnhS8buMXOCeQw\%3D\%3D\&crl=c

Adams, R., Kewell, B., \& Parry, G. (2018). Blockchain for good? Digital ledger technology and sustainable development goals. In W. Leal Filho, R. Marans, \& J. Callewaert (Eds.), Handbook of sustainability and social science research (pp. 127-140). Springer. https://doi.org/10.1007/978-3-319-67122-2_7

Borgatti, S. P., \& Li, X. (2009). On social network analysis in a supply chain context. Journal of Supply Chain Management, 45(2), 5-22. https://doi.org/10.1111/j.1745-493X.2009.03166.x

Busse, C., Meinlschmidt, J., \& Foerstl, K. (2017). Managing information processing needs in global supply chains: a prerequisite to sustainable supply chain management. Journal of Supply Chain Management, 53(1), 87-113. https://doi.org/10.1111/jscm.12129

Carter, C. R., Rogers, D. S., \& Choi, T. Y. (2015). Toward the theory of the supply chain. Journal of Supply Chain Management, 51(2), 89-97. https://doi.org/10.1111/jscm.12073

Choi, T.-M., Feng, L., \& Li, R. (2020). Information disclosure structure in supply chains with rental service platforms in the blockchain technology era. International Journal of Production Economics, 221, 107473. https://doi.org/10.1016/j.ijpe.2019.08.008

de Sousa Jabbour, A. B. L., Jabbour, C. J. C., Foropon, C., \& Filho, M. G. (2018). When titans meet Can industry 4.0 revolutionise the environmentally-sustainable manufacturing wave? The role of critical success factors. Technological Forecasting and Social Change, 132, 18-25. https://doi.org/10.1016/j.techfore.2018.01.017

Dolgui, A., Ivanov, D., \& Sokolov, B. (2018). Ripple effect in the supply chain: an analysis and recent literature. International Journal of Production Research, 56(1-2), 414-430. https://doi.org/10.1080/00207543.2017.1387680

Ellis, S. C., Shockley, J., \& Henry, R. M. (2011). Making sense of supple disruption risk research: a conceptual framework grounded in enactment theory. Journal of Supply Chain Management, 47(2), 65-96. https://doi.org/10.1111/j.1745-493X.2011.03217.x

Eyal, I. (2017). Blockchain technology: transforming libertarian cryptocurrency dreams to finance and banking realities. Computer, 50(9), 38-49. https://doi.org/10.1109/MC.2017.3571042

Fahimnia, B., Sarkis, J., \& Davarzani, H. (2015). Green supply chain management: A review and bibliometric analysis. International Journal of Production Economics, 162, 101-114. https://doi.org/10.1016/j.ijpe.2015.01.003

Fanning, K., \& Centers, D. P. (2016). Blockchain and its coming impact on financial services. Journal of Corporate Accounting \& Finance, 27(5), 53-57. https://doi.org/10.1002/jcaf.22179

Flynn, B. B., Koufteros, X., \& Lu, G. (2016). On theory in supply chain uncertainty and its implications for supply chain integration. Journal of Supply Chain Management, 52(3), 3-27. https://doi.org/10.1111/jscm.12106

Govindan, K., \& Hasanagic, M. (2018). A systematic review on drivers, barriers, and practices towards circular economy: a supply chain perspective. International Journal of Production Research, 56(1-2), 278-311. https://doi.org/10.1080/00207543.2017.1402141 
Gray, J. V., Skowronski, K., Esenduran, G., \& Johnny Rungtusanatham, M. (2013). The reshoring phenomenon: What supply chain academics ought to know and should do. Journal of Supply Chain Management, 49(2), 27-33. https://doi.org/10.1111/jscm.12012

Hendalianpour, A. (2020). Optimal lot-size and price of perishable goods: a novel game-theoretic model using double interval grey numbers. Computers \& Industrial Engineering, 149, 106780. https://doi.org/10.1016/j.cie.2020.106780

Hendalianpour, A., Fakhrabadi, M., Sangari, M. S., \& Razmi, J. (2020b). A combined benders decomposition and lagrangian relaxation algorithm for optimizing a multi-product, multi-level Omni-channel distribution system. Scientia Iranica (in press). https://doi.org/10.24200/sci.2020.53644.3349

Hendalianpour, A., Fakhrabadi, M., Zhang, X., Feylizadeh, M. R., Gheisari, M., Liu, P., \& Ashktorab, N. (2019). Hybrid model of IVFRN-BWM and robust goal programming in agile and flexible supply chain, a case study: automobile industry. IEEE Access, 7, 71481-71492.

https://doi.org/10.1109/ACCESS.2019.2915309

Hendalianpour, A., Hamzehlou, M., Feylizadeh, M. R., Xie, N., \& Shakerizadeh, M. H. (2020a). Coordination and competition in two-echelon supply chain using grey revenue-sharing contracts. Grey Systems: Theory and Application (in press). https://doi.org/10.1108/GS-04-2020-0056

Hofmann, E., Strewe, U. M., \& Bosia, N. (2018). Supply chain finance and blockchain technology: the case of reverse securitisation. Springer. https://books.google.com/books?hl=en\&lr=\&id=tLIvDwAA QBAJ\&oi=fnd\&pg $=$ PR5\&dq=Hofmann, + E.,+ U. + M. + Strewe,+ and + N. + Bosia.$+2017 .+$ Supply + Cha in+Finance+and+Blockchain+Technology:+The+Case+of+Reverse+Securitisation.+Cham:+Sprin ger.\&ots=kVXZXUAurW\&sig=wxvx32gi-tb794A

Ivanov, D., Dolgui, A., \& Sokolov, B. (2019). The impact of digital technology and Industry 4.0 on the ripple effect and supply chain risk analytics. International Journal of Production Research, 57(3), 829-846. https://doi.org/10.1080/00207543.2018.1488086

Kass, R. E., Gilks, W. R., Richardson, S., \& Spiegelhalter, D. J. (1997). Markov chain Monte Carlo in practice. Journal of the American Statistical Association, 92(440), 1645-1646. https://doi.org/10.2307/2965438

Kasten, J. (2019). Blockchain application: the dairy supply chain. Journal of Supply Chain Management Systems, 8(1), 45-54. http://search.ebscohost.com/login.aspx?direct=true\&profile=ehost\&scope=si te\&authtype $=$ crawler\&jrnl $=22771387 \& A N=136630613 \& \mathrm{~h}=\mathrm{VMq} \% 2 \mathrm{BA} 6 \mathrm{q} 3 \mathrm{EQUOIRGqZ}$ roWvm0 dlz2WkrSUuCegNFf\%2FvdqDht6W\%2B4KAA\%2BWfKeEh9s\%2BeRkV95UkksRdEv3q9oxquGA $\% 3 \mathrm{D} \% 3 \mathrm{D} \& \mathrm{crl}=\mathrm{c}$

Manupati, V. K., Schoenherr, T., Ramkumar, M., Wagner, S. M., Pabba, S. K., \& Inder Raj Singh, R. (2020). A blockchain-based approach for a multi-echelon sustainable supply chain. International Journal of Production Research, 58(7), 2222-2241. https://doi.org/10.1080/00207543.2019.1683248

Mcivor, R. (2013). Understanding the manufacturing location decision: The case for the transaction cost and capability perspectives. Journal of Supply Chain Management, 49(2), 23-26. https://doi.org/10.1111/jscm.12010

Mohammadi, M., \& Rezaei, J. (2019). Bayesian best-worst method: A probabilistic group decision making model. Omega, 96, 102075. https://doi.org/10.1016/j.omega.2019.06.001

Ølnes, S., Ubacht, J., \& Janssen, M. (2017). Blockchain in government: Benefits and implications of distributed ledger technology for information sharing. Government Information Quarterly, 34(3), 355-364. https://doi.org/10.1016/j.giq.2017.09.007

Plummer, M. (2004). JAGS: Just another Gibbs sampler. http://www3.jouy.inra.fr/miaj/public/matrisq/ Contacts/applibugs.07_11_08.plummer.pdf

Rajeev, A., Pati, R. K., Padhi, S. S., \& Govindan, K. (2017). Evolution of sustainability in supply chain management: A literature review. Journal of Cleaner Production, 162, 299-314.

https://doi.org/10.1016/j.jclepro.2017.05.026 
Rezaei, J. (2016). Best-worst multi-criteria decision-making method: Some properties and a linear model. Omega, 64, 126-130. https://doi.org/10.1016/j.omega.2015.12.001

Saberi, S., Kouhizadeh, M., \& Sarkis, J. (2018). Blockchain technology: A panacea or pariah for resources conservation and recycling? Resources, Conservation and Recycling, 130, 80-81. https://doi.org/10.1016/j.resconrec.2017.11.020

Saberi, S., Kouhizadeh, M., Sarkis, J., \& Shen, L. (2019a). Blockchain technology and its relationships to sustainable supply chain management. International Journal of Production Research, 57(7), 21172135. https://doi.org/10.1080/00207543.2018.1533261

Saberi, S., Kouhizadeh, M., Sarkis, J., \& Shen, L. (2019b). Blockchain technology and its relationships to sustainable supply chain management. International Journal of Production Research, 57(7), 21172135. https://doi.org/10.1080/00207543.2018.1533261

Salo, A. A., \& Hämäläinen, R. P. (1997). On the measurement of preferences in the analytic hierarchy process. Journal of Multi-Criteria Decision Analysis, 6(6), 309-319.

https://doi.org/10.1002/(SICI)1099-1360(199711)6:6<309::AID-MCDA163>3.0.CO;2-2

Schmidt, C. G., \& Wagner, S. M. (2019). Blockchain and supply chain relations: A transaction cost theory perspective. Journal of Purchasing and Supply Management, 25(4), 100552. https://doi.org/10.1016/j.pursup.2019.100552

Schniederjans, D. G., Curado, C., \& Khalajhedayati, M. (2020). Supply chain digitisation trends: An integration of knowledge management. International Journal of Production Economics, 220, 107439. https://doi.org/10.1016/j.ijpe.2019.07.012

Skilton, P. F., \& Robinson, J. L. (2009). Traceability and normal accident theory: How does supply network complexity influence the traceability of adverse events? Journal of Supply Chain Management, 45(3), 40-53. https://doi.org/10.1111/j.1745-493X.2009.03170.x

Tian, F. (2012). An information system for food safety monitoring in supply chains based on HACCP, blockchain and internet of things [Doctoral dissertation]. WU Vienna University of Economics and Business. http://epub.wu.ac.at/

Tian, F. (2016). An agri-food supply chain traceability system for China based on RFID \& blockchain technology. In 13th International Conference on Service Systems and Service Management (ICSSSM). Institute of Electrical and Electronics Engineers Inc. https://doi.org/10.1109/ICSSSM.2016.7538424

Tokar, T., \& Swink, M. (2019). Public policy and supply chain management: using shared foundational principles to improve formulation, implementation, and evaluation. Journal of Supply Chain Management, 55(2), 68-79. https://doi.org/10.1111/jscm.12190

Underwood, S. (2016). Blockchain beyond bitcoin. Communications of the ACM, 59(11), 15-17. https://doi.org/10.1145/2994581

Wang, Y., Singgih, M., Wang, J., \& Rit, M. (2019). Making sense of blockchain technology: How will it transform supply chains? International Journal of Production Economics, 211, 221-236. https://doi.org/10.1016/j.ijpe.2019.02.002

Yatsalo, B. I., \& Martinez, L. (2018). Fuzzy rank acceptability analysis: A confidence measure of ranking fuzzy numbers. IEEE Transactions on Fuzzy Systems, 26(6), 3579-3593. https://doi.org/10.1109/TFUZZ.2018.2838063

Zhao, G., Liu, S., Lopez, C., Lu, H., Elgueta, S., Chen, H., \& Boshkoska, B. M. (2019). Blockchain technology in agri-food value chain management: A synthesis of applications, challenges and future research directions. Computers in Industry, 109, 83-99. https://doi.org/10.1016/j.compind.2019.04.002

Zijm, H., Knofius, N., \& van der Heijden, M. (2019). Additive Manufacturing and Its Impact on the Supply Chain. In H. Zijm, M. Klumpp, A. Regattieri, \& S. Heragu (Eds.), Operations, logistics and supply chain management (pp. 521-543). Springer. https://doi.org/10.1007/978-3-319-92447-2_23 